Abanico Agroforestal. Enero-Diciembre 2021; 1-18. http://dx.doi.org/10.37114/abaagrof/2021.1 Artículo Original. Recibido: 04/12/2020. Aceptado: 14/01/2021. Publicado: 30/01/2021. Clave:2020-15.

\title{
Estado del arte de la producción lechera mexicana
}

\author{
State of the art of mexican milk production
}

\author{
Martínez-Alba María1 ID , Molina-Morejón Víctor ${ }^{2}$ ID , García-Munguía Carlos ${ }^{3}$ ID , Díaz-

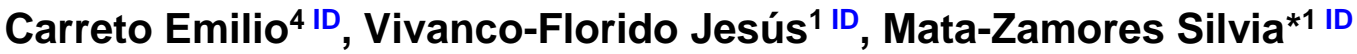

${ }^{1}$ Centro de Ciencias Económico Administrativas de la Universidad Autónoma de Aguascalientes, México. C.P. 20131. ' $F$ Facultad de Contaduría y Administración en la Universidad Autónoma de Coahuila, México. C.P. 25710. ${ }^{3}$ División de Ciencias de la Vida en la Universidad de Guanajuato, México. C.P. 36500. ${ }^{4}$ Gerente de Suministros Lácteos en Nestlé México. C.P. $47425 .{ }^{1 *}$ Autor por correspondencia: Centro de Ciencias Económico Administrativas de la Universidad Autónoma de Aguascalientes, México. al139740@edu.uaa.mx.mtzalba@live.com.mx, vmolinaa2005@yahoo.com.mx, cagamu@hotmail.com, emilio.diaz@mx.nestle.com, doctorsalvatore@yahoo.com.mx

\section{Resumen}

En la producción mundial lechera, México ocupó la octava posición (2017 - 2019), mientras que, en la compra de leche en polvo posee el primer lugar con el 11.2\% de las importaciones globales (SIAP, 2019). Considerando así, que la competitividad del sector lechero en México depende de la mejora continua de la productividad a largo plazo. El objetivo del presente estudio fue evaluar el contexto actual de la producción lechera mexicana, a través de una revisión bibliográfica y por medio de una correlación de Pearson $(\mathrm{P}<0.01)$ de las variables cuantitativas: volumen, cabezas de ganado, precio constante, consumo per cápita e importaciones de leche en polvo. Observando que las importaciones de leche en polvo mantienen una alta correlación con la variable volumen (.883), entendiéndose así que a pesar de que la producción se mantenga al alza, no es suficiente para abastecer la demanda nacional.

Palabras clave: leche, volumen, precio, importación, México.

\begin{abstract}
In world milk production, Mexico ranks eighth (2017 - 2019), while in the purchase of powdered milk it holds first place with $11.2 \%$ of global imports (SIAP, 2019). Thus, the competitiveness of the dairy sector in Mexico depends on the continuous improvement of productivity in the long term. The objective of this study was to evaluate the current context of Mexican milk production, through a bibliographic review and by means of a Pearson correlation $(\mathrm{P}<0.01)$ of the quantitative variables:volume, heads of cattle, constant price, per capita consumption and imports of powdered milk. Observing that powdered milk imports maintain a high correlation with the volume variable $(.883)$, it is understood that even though production remains high, it is not enough to supply the national demand.
\end{abstract}

Keywords: volume, yield, price, import, Mexico. 


\section{INTRODUCCIÓN}

La Organización Mundial de Sanidad Animal (OIE) en el año 2013 definió las unidades de producción lechera como un sistema comercial de producción de ganado cuya finalidad implica la crianza, reproducción y administración del ganado con designio a la producción de leche; mencionando que los principales sistemas establecidos son de tipo integrado, intensivo y extensivo (OIE, 2013; Herrera et al., 2011). Además de considerar que el trabajo en este sector cuenta con características específicas; pues está condicionado a esfuerzo físico en la manipulación del ganado, aislamiento, separación social y dependencia del medio natural (Main, 2002).

En el año 2018 la Unión europea ocupó el primer lugar en la producción de leche de bovino con una producción de 154.8 millones de toneladas, lo que representó el 30.6\% de producción a nivel mundial (figura 1). Además de observarse que, en el mismo año, Estados Unidos de América fue el segundo productor más importante con una producción de 98.8 millones de toneladas, representando el $19.6 \%$ de la producción mundial (FIRA, 2019), y con un estimado de 9.3 millones de cabezas de ganado; es considerado como el país con el mayor nivel de productividad, con 10.5 toneladas de leche por vaca/año. Dairy Farming in the United States (DFUS) adjudica su alta productividad al buen manejo sanitario e implementación de dietas nutritivas y balanceadas del ganado, praderas con un manejo agronómico, así como instalaciones que aseguran el bienestar animal (DFUS, 2020). De igual manera, se puede observar (figura 1) que países como Polonia, Reino Unido, Alemania, Francia y Holanda logran destacar, gracias a un hato lechero que supera los cuatro millones de cabezas; con una productividad aproximada de 6.6 toneladas de leche vaca/año (FIRA, 2019).

Durante el mismo año (2018), la India se posicionó en el tercer lugar en la producción lechera mundial, con 78 millones de toneladas (figura 1); donde en este país figuran unidades de producción lecheras con hasta cinco cabezas de ganado y con un poco inversión. Siendo así que India también posee el mayor hato lechero bovino, el cual se estima en 58.5 millones de cabezas; sin embargo, su productividad es la más baja entre los principales productores, con 1.3 toneladas de leche vaca/año (FIRA, 2019; Hemme et al., 2003). Comparando a México con los países anteriormente mencionados y en el mismo año de producción, se observa que ocupó la octava posición (figura 1), con una aportación del $2.4 \%$ de la producción total mundial. 


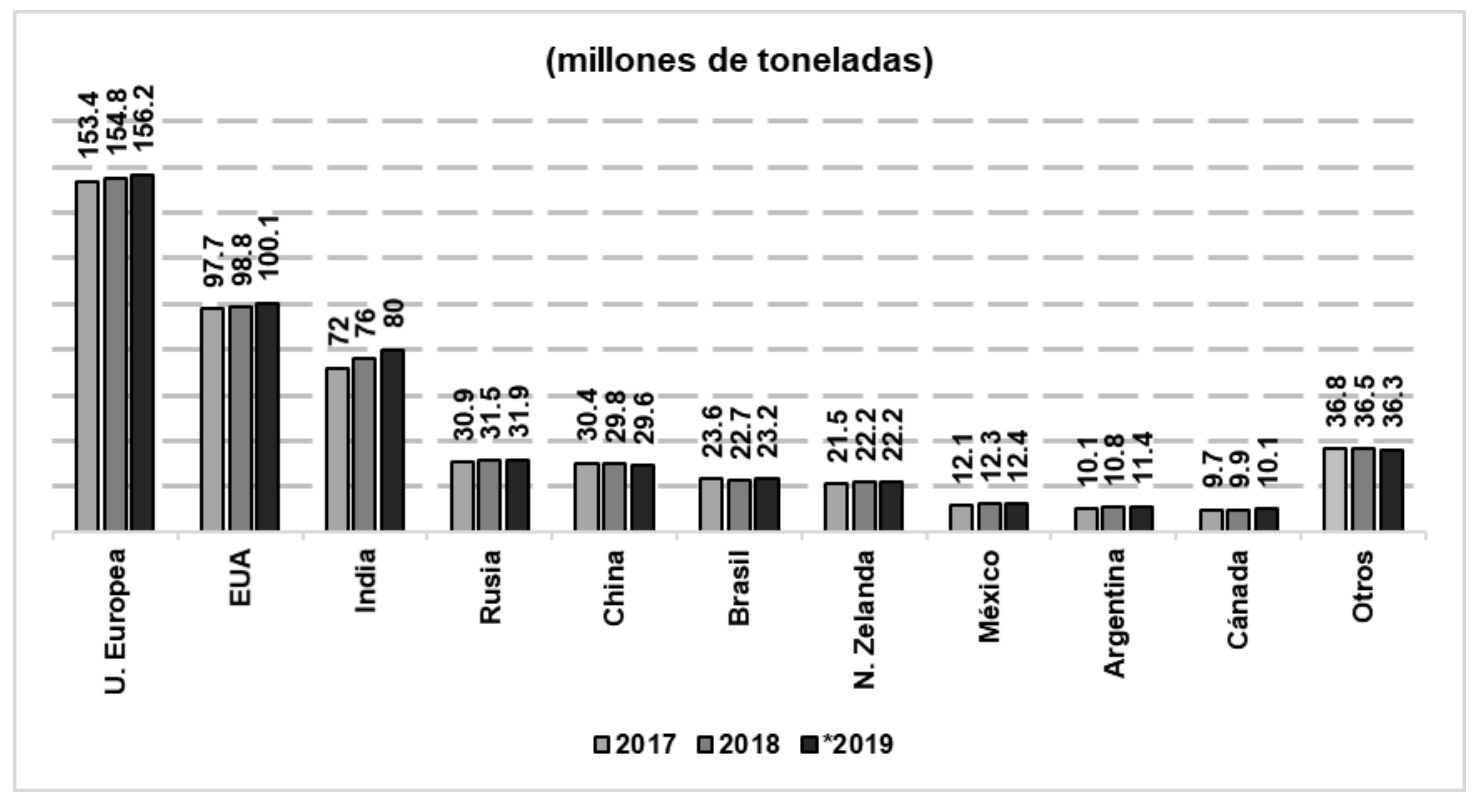

Figura 1. Principales productores de leche en el ámbito internacional

Fuente: FIRA con datos de la USDA, 2019.

Si bien la producción lechera internacionalmente se logra a partir de diferentes sistemas de producción; donde países como Australia, Nueva Zelanda, Argentina y Uruguay registran sobrantes en su productividad debido a la alta disponibilidad de recursos naturales, lo cual les permite producir más y a menor costo (Santibáñez y Sánchez, 2009); en México su producción se puede ver condicionada por la heterogeneidad de las unidades de producción animal (número de cabezas de ganado, adopción de tecnología, reproducción, alimentación, comercialización, etc.) (Camacho et al., 2017a; López et al., 2014).

Recordando que, en el ámbito mundial, la leche y sus derivados se consideran bienes básicos (Vera, 2020), cotizando en dólares y su precio fluctúa en los mercados continuamente (Doporto y Michelena, 2011). Es por esto que, algunos países han optado por asegurar la producción de leche como recurso para proveer la demanda nacional, mientras que otros han preferido las importaciones (Sraïri et al., 2013). Siendo importante recordar, que desde tiempo atrás, la producción lechera ha presentado una crisis de rentabilidad debido a los bajos precios del producto y las grandes inversiones en materias primas (Próspero et al., 2013).

La Organización de las Naciones Unidas para la Alimentación y la Agricultura (FAO) en conjunto con la Organización para la Cooperación y el Desarrollo Económicos (OCDE), mencionan que en diversos lugares del mundo se ha propuesto que entre las grandes y pequeñas unidades de producción lecheras existen brechas de productividad, entendidas como las diferencias profundas entre la producción promedio obtenida de 
leche por vaca en el hato, por hectárea de terreno laborable, por salario laborado o por año, entre otras variables (FAO et al., 2012; Escudero et al., 2012).

Dichas brechas de productividad se reflejan en caminos de rentabilidad económica (Hanson et al., 2013; Segura y Lozano, 2015), autores como Aragon y Rubio (2005) las definen como un indicador que cuantifica las relaciones de intercambio de las unidades de producción lecheras con el mercado, reflejando el equilibrio entre inversiones como la mano de obra y los insumos (Jiménez et al., 2014), con el precio de los productos pagados al participante en la cadena de producción lechera (Qüesta et al., 2016). Donde las unidades de producción con hatos grandes disminuyen sus gastos de operación unitarios al distribuir los costos fijos de instalaciones, equipo de ordeño y servicios de asistencia técnica, en un volumen de producción muy grande, lo cual incrementa sus ganancias y las posibilidades económicas de implementar mejoras en la calidad del proceso (Hanson et al., 2013; Segura y Lozano, 2015). Sin embargo, en las unidades de producción animal con un mínimo de cabezas de ganado, pueden existir brechas en el alcance del potencial técnico de producción y, por lo tanto, en la rentabilidad económica y el desarrollo tecnológico (Bacco et al., 2013).

Ahora bien, es importante destacar que la rentabilidad económica es sensible a la disponibilidad de recursos agroecológicos (Mamián et al., 2016), distorsiones del mercado por subsidios, acaparamiento, control nacional e internacional de los precios de los lácteos, insumos y sustitutos de leche (Escudero et al., 2013; Hemme et al., 2014).

En México, la productividad de la ganadería lechera como actividad primaria, y sus conexiones con los eslabones de industrialización y comercialización de la leche fluida y sus derivados, es un tema de relevancia para la economía nacional (Núñez, 2016). De manera histórica, se observa una brecha importante entre la producción nacional y la demanda de leche; ese déficit para cubrir la demanda interna es una de las razones por las cuales el país ha ocupado el primer lugar como importador de leche en polvo en el mundo (Loera y Banda, 2017; Salomón y Ramírez, 2018). Es por ello que el sector lechero mexicano requiere de propuestas viables y soluciones prácticas que les permita a los productores participar de manera exitosa en la producción nacional, compitiendo en el mercado, con una mejora en sus ingresos; pero protegiendo la biodiversidad y su riqueza cultural (Garay et al., 2011). Además de hacer hincapié en la calidad y administración; pues se considera que estos elementos condicionan la transición de las empresas agropecuarias hacia nuevas formas de organización competitiva (Arce y Martínez, 2007). Pues la dirección exitosa de unidades de producción agropecuarias necesita conocer, organizar, regular y accionar en forma sistémica (Guevara et al., 2004).

Siendo así el objetivo del presente estudio, evaluar el contexto actual de la producción lechera mexicana, desde las perspectivas de producción y competitividad de las últimas 
dos décadas; esto a través de las variables cuantitativas: volumen, cabezas de ganado, precio constante, consumo per cápita e importaciones de leche en polvo.

\section{MATERIAL Y MÉTODOS}

Fuentes de información: Para el presente estudio, los datos de las variables volumen, cabezas de ganado, precio nominal, consumo per cápita e importaciones de leche en polvo fueron obtenidos del Sistema de Información Agroalimentaria y Pesquera (SIAP), tomando como referencia los datos generados entre 2000-2019 del subsector de bovinos lecheros.

Metodología empleada: Se realizó un análisis de correlación con la finalidad de examinar la dirección y la fuerza de la asociación entre las variables: volumen, cabezas de ganado, precio constante, consumo per cápita e importaciones de leche en polvo. El estimador muestral utilizado para evaluar fue el coeficiente de correlación de Pearson (r) con el programa estadístico SPSS versión 27, y la interpretación de los resultados se realizó con base en los criterios de Hinkle et al. (2003). Previo al análisis de correlación, se realizó la deflactación de los precios de la leche por litro.

Deflactación de precios de la leche: Se refiere a eliminar de un valor monetario los efectos producidos por los cambios en los precios (inflación o deflación) (García et al., 2006). Convirtiendo una magnitud medida en términos nominales en otra expresada en términos reales.

$$
\mathrm{P}=\frac{\text { Precio nóminal }}{I P} * 100
$$

Donde:

$\mathrm{P}=$ Precios reales

IP = índice de Precio del año base $2010=100$.

\section{RESULTADOS Y DISCUSIÓN}

El objetivo del presente estudio fue determinar y analizar la correlación entre las variables cuantitativas: los resultados obtenidos se puede observar la media y desviación de cada una de las variables (tabla 1), así como su correlación (tabla 2). A continuación, se analiza cada una de las variables de estudio.

Tabla 1. Estadísticos descriptivos

\begin{tabular}{lr|r|r} 
& \multicolumn{1}{c|}{ Media } & \multicolumn{1}{c}{ Desviación } & \multicolumn{1}{c}{ N } \\
\hline VOLUMEN & 10650162.40 & 866038.473 & 20 \\
\hline PRECIO CONSTANTE & 4.438000 & 1.8423572 & 20 \\
\hline CONSUMO PER CÁPITA & 118.385 & 6.5094 & 20 \\
\hline CABEZAS DE GANADO & 2335955.90 & 143903.375 & 20 \\
$\begin{array}{l}\text { IMPORTACIÓN LECHE EN } \\
\text { POLVO }\end{array}$ & 217100.00 & 70928.355 & 20 \\
\hline
\end{tabular}


Tabla 2. Correlaciones

\begin{tabular}{|c|c|c|c|c|c|c|}
\hline & & VOLUMEN & $\begin{array}{c}\text { PRECIO } \\
\text { CONSTANTE }\end{array}$ & $\begin{array}{l}\text { CONSUMO } \\
\text { PER CÁPITA }\end{array}$ & $\begin{array}{c}\text { CABEZAS } \\
\text { DE } \\
\text { GANADO }\end{array}$ & $\begin{array}{l}\text { IMPORTACIÓN } \\
\text { LECHE EN } \\
\text { POLVO }\end{array}$ \\
\hline \multirow[t]{5}{*}{ VOLUMEN } & $\begin{array}{l}\text { Correlación de } \\
\text { Pearson }\end{array}$ & 1 & $.770^{\star \star}$ & -.269 & $.985^{\star \star}$ & $.883^{\star *}$ \\
\hline & Sig. (bilateral) & & .000 & .252 & .000 & .000 \\
\hline & $\begin{array}{l}\text { Suma de } \\
\text { cuadrados y } \\
\text { productos } \\
\text { vectoriales }\end{array}$ & $1,425 \mathrm{E}+13$ & 23337635,61 & $-28798567,3$ & $2,332 \mathrm{E}+12$ & $1,031 \mathrm{E}+12$ \\
\hline & Covarianza & $7,500 \mathrm{E}+11$ & 1228296.611 & 1515714.067 & $1,227 \mathrm{E}+11$ & $5,425 \mathrm{E}+10$ \\
\hline & $\mathrm{N}$ & 20 & 20 & 20 & 20 & 20 \\
\hline \multirow[t]{5}{*}{$\begin{array}{l}\text { PRECIO } \\
\text { CONSTANTE }\end{array}$} & $\begin{array}{l}\text { Correlación de } \\
\text { Pearson }\end{array}$ & $.770^{\star \star}$ & 1 & -.414 & $.783^{\star \star}$ & $.628^{\star *}$ \\
\hline & Sig. (bilateral) & .000 & & .069 & .000 & .003 \\
\hline & $\begin{array}{l}\text { Suma de } \\
\text { cuadrados y } \\
\text { productos } \\
\text { vectoriales }\end{array}$ & 23337635,61 & 64.491 & -94.422 & 3946400.506 & 1559544.00 \\
\hline & Covarianza & 1228296.611 & 3.394 & -4.970 & 207705.290 & 82081.263 \\
\hline & $\mathrm{N}$ & 20 & 20 & 20 & 20 & 20 \\
\hline \multirow[t]{5}{*}{$\begin{array}{l}\text { CONSUMO } \\
\text { PER CÁPITA }\end{array}$} & $\begin{array}{l}\text { Correlación de } \\
\text { Pearson }\end{array}$ & -.269 & -.414 & 1 & -.345 & -.071 \\
\hline & Sig. (bilateral) & .252 & .069 & & .136 & .765 \\
\hline & $\begin{array}{l}\text { Suma de } \\
\text { cuadrados y } \\
\text { productos } \\
\text { vectoriales }\end{array}$ & $-28798567,3$ & -94.422 & 805.066 & 6143255.830 & -626670.000 \\
\hline & Covarianza & -1515714.067 & -4.970 & 42.372 & -323329.254 & -32982.632 \\
\hline & $\mathrm{N}$ & 20 & 20 & 20 & 20 & 20 \\
\hline \multirow[t]{5}{*}{$\begin{array}{l}\text { CABEZAS DE } \\
\text { GANADO }\end{array}$} & $\begin{array}{l}\text { Correlación de } \\
\text { Pearson }\end{array}$ & $.985^{\star \star}$ & $.783^{\star \star}$ & -.345 & 1 & $.822^{* \star}$ \\
\hline & Sig. (bilateral) & .000 & .000 & .136 & & .000 \\
\hline & $\begin{array}{l}\text { Suma de } \\
\text { cuadrados y } \\
\text { productos } \\
\text { vectoriales }\end{array}$ & $2,332 \mathrm{E}+12$ & 3946400.506 & 6143255.830 & $3,935 \mathrm{E}+11$ & $1,595 E+11$ \\
\hline & Covarianza & $1,227 \mathrm{E}+11$ & 207705.290 & -323329.254 & $2,971 E+10$ & 8394496905 \\
\hline & $\mathrm{N}$ & 20 & 20 & 20 & 20 & 20 \\
\hline \multirow{5}{*}{$\begin{array}{l}\text { IMPORTACIÓN } \\
\text { DE LECHE EN } \\
\text { POLVO }\end{array}$} & $\begin{array}{l}\text { Correlación de } \\
\text { Pearson }\end{array}$ & $.883^{\star *}$ & $.628^{\star \star}$ & -.071 & $.822^{\star \star}$ & 1 \\
\hline & Sig. (bilateral) & .000 & .003 & .765 & .000 & \\
\hline & $\begin{array}{l}\text { Suma de } \\
\text { cuadrados y } \\
\text { productos } \\
\text { vectoriales }\end{array}$ & $1,031 \mathrm{E}+12$ & 1559533.000 & -626670.000 & $1,595 \mathrm{E}+11$ & $9,559 \mathrm{E}+10$ \\
\hline & Covarianza & $5,425 \mathrm{E}+10$ & 82081.263 & -32982.632 & 8394496905 & 5030831579 \\
\hline & $\mathrm{N}$ & 20 & 20 & 20 & 20 & 20 \\
\hline
\end{tabular}




\section{Volumen - Cabezas de Ganado}

Donde datos del Servicio de Información Agroalimentaria y Pesquera (SIAP), menciona que la apreciación de producción mundial de leche para el año 2019 posicionaba a la Unión Europea como principal productor lechero; mientras que México ocupaba el octavo lugar (SIAP, 2019). En contraste con la Organización de las Naciones Unidas para la Alimentación y la Agricultura (FAO) (2010), quien considera que los países con mayores pérdidas en leche son Rusia, México, China, Indonesia, Italia y Argelia; donde México es considerado uno de los países con menor productividad de leche por vaca, sólo superado por la India y Brasil.

Tomando como referencia la información anteriormente mencionada y al realizar el análisis de correlación de las variables volumen y número de cabezas de ganado de las últimas dos décadas en México, se arroja como resultado una alta correlación (.985) entre ambas variables (figura 2).

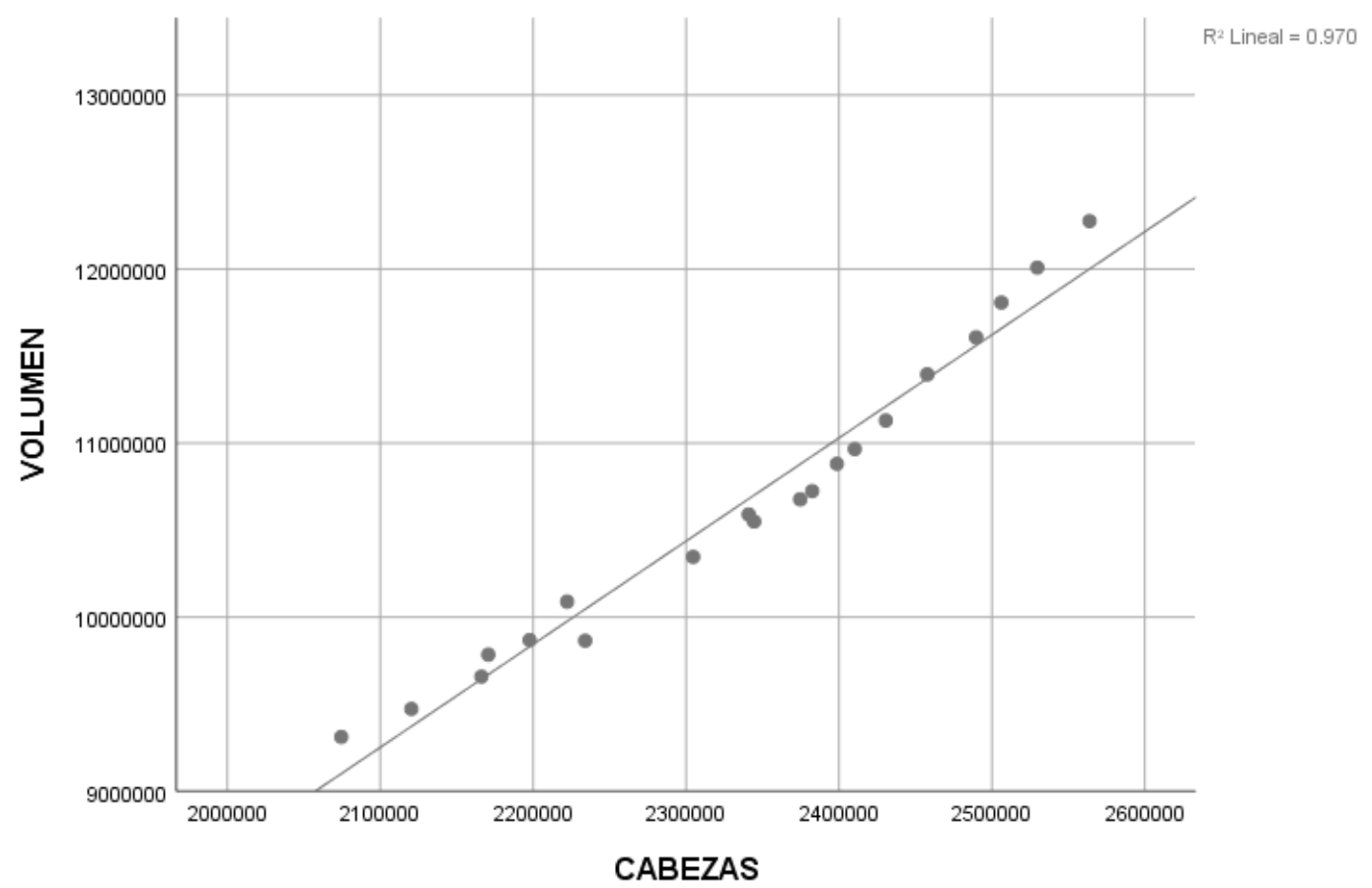

Fuente: Elaboración propia, 2020.

Figura 2. Dispersión simple con ajuste de línea de las variables Volumen por Cabezas de Ganado, ciclo 2000-2019

Lo cual es interpretado como un déficit en la productividad lechera en México, pues lo ideal es que el número de cabezas de ganado fuera decreciente o de lo contrario permanente en los últimos años; entendiéndose así que el ganado cuenta con un buen 
rendimiento. Por lo cual se pone en duda la rentabilidad de las unidades de producción lecheras mexicanas.

Siendo importante considerar que México cuenta con una producción lechera muy variada de una región a otra, así como factores en la adopción de tecnologías, el contexto agroecológico y socioeconómico, además de las condiciones climáticas y culturales de las comunidades (Camacho et al., 2017b; SIAP, 2019). Donde el éxito de los sistemas de producción agropecuarios dependerá de los recursos disponibles, productividad e ingresos, tecnología aplicada y producto generado (Barrios y Olivera, 2013).

\section{Precio constante}

EI SIAP reportó en el año 2019 que el valor de la producción de leche de bovino en México durante 2018 superó los 71 mil millones de pesos. Con una producción diaria promedio de 32.9 millones de litros de leche. Además de resaltar que la producción de leche en México es mayor durante el verano, debido a que el temporal aumenta la disponibilidad de forrajes (FIRA, 2019).

Durante el estudio, se realizó la deflactación del precio corriente tomando como precio base el año 2010, obteniendo así el precio constante por litro de leche pagado al productor en el ciclo 2000-2019 (figura 3). Observando una baja importante en el año 2017, para luego verse recuperado en el 2019.

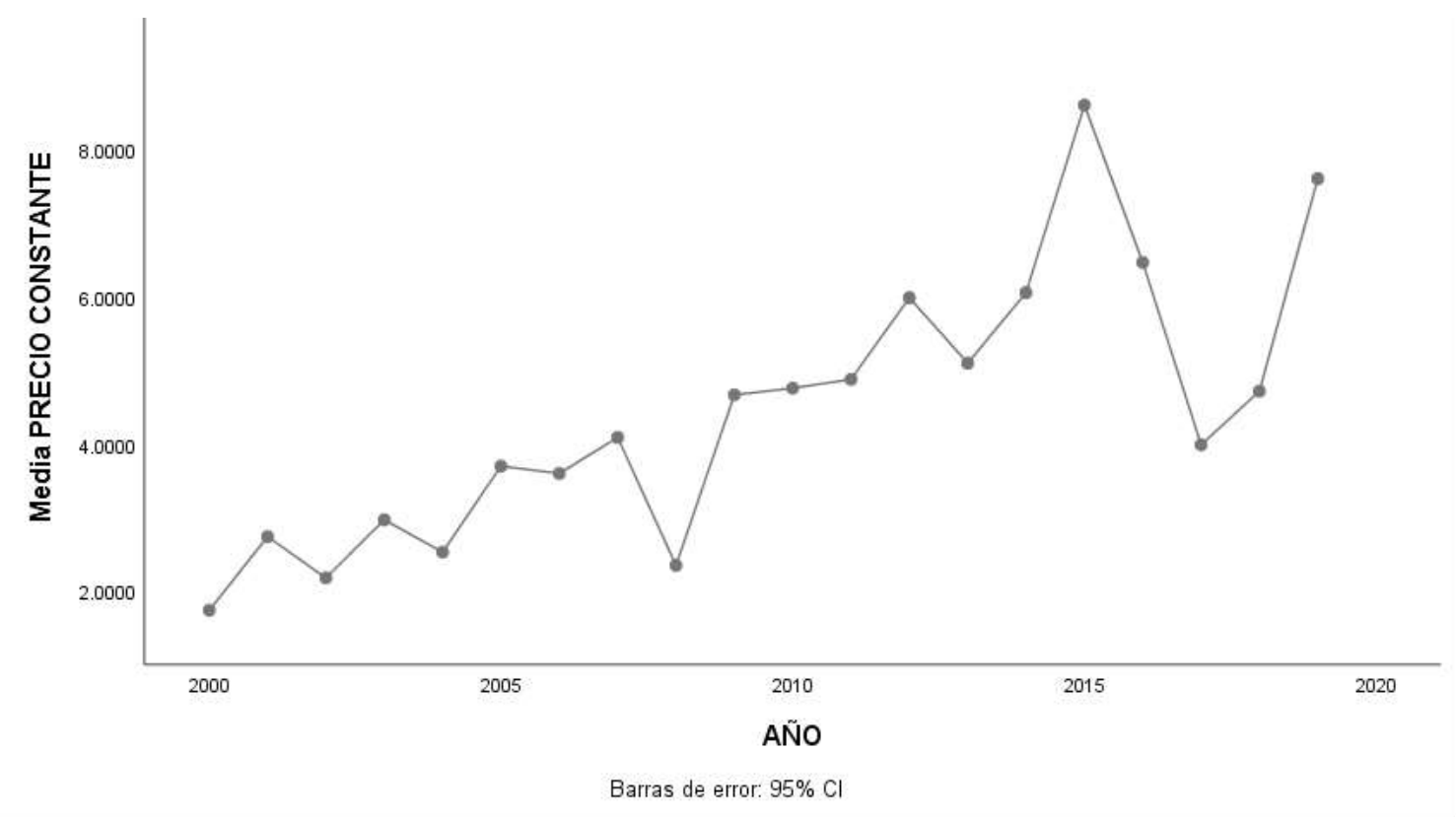

Fuente: Elaboración propia, con datos del SIAP (2020).

Figura 3. Barras de error simples de media de Precio Constante por año (2000-2019) 
Si tomamos como referencia el año 2017, es importante considerar que el aumento de los precios de los combustibles y de los alimentos fue visible en el comportamiento de la inflación no subyacente que se disparó de $3.1 \%$ en 2016 a $12.6 \%$ en 2017 , como resultado de una aceleración en todos sus componentes: agropecuarios (9.8\%), energéticos (17.7\%) y tarifas autorizadas por el gobierno (8.4\%) (Cordera et al., 2017).

Ahora bien, al realizar el análisis de correlación de Pearson y tomando como referencia a Hinkle et al. (2003) para su interpretación, la variable consumo per cápita obtuvo una ausencia de correlación (-.414) con el precio constante. Lo cual indica que, aunque el consumo se mantenga al alza, este no influye con el precio constante pagado al productor. Mientras que en la variable importaciones de leche en polvo se obtuvo una correlación moderada (.628). Lo cual coincide con lo expuesto por Robledo (2018) y Cervantes y Cesín (2019); quienes concluye de manera preliminar que el precio de la leche que se paga al productor en México continuará siendo bajo debido al alza de la importación de leche en polvo que desplaza a la producción nacional. A su vez, autores como Ramírez et al. (2010) y Davalos y Villegas (2005) mencionan que en el marco de la renegociación del Tratado de Libre Comercio de América del Norte (TLCAN), la leche es uno de los puntos perceptibles para México, pues la vulnerabilidad de este mercado se relaciona directamente con los precios. Además de considerar que actualmente el termino de "sostenibilidad" mantiene un impacto en la rentabilidad de las unidades de producción animal; pues en una investigación realizada por Olivera et al. (2018) concluyen que los productores pecuarios no conocen ni dimensionan la trascendencia para la productividad y rentabilidad que tendría la aplicación de herramientas económicas, sociales y medioambientales; implicando así el término "desarrollo sostenible" (Girón, 2016). Demostrando que la productividad como la rentabilidad de las unidades de producción lecheras, así como la calidad de vida de los productores, se verán fortalecidas por la aplicación de sostenibilidad (Zarate et al., 2010).

\section{Consumo per cápita}

La Dirección de Investigación y Evaluación Económica Sectorial de FIRA, a través del informe de leche y lácteos 2019, realizó un compendio de información relevante desde el contexto nacional e internacional de la producción lechera. Donde informa que acorde con datos del Departamento de Agricultura de Estados Unidos (USDA), en el 2018 el consumo mundial de leche fluida logro un máximo histórico de 605.3 millones de toneladas. Donde el uso industrial representa el mayor consumo con el $70 \%$ en el mismo año, ya sea para agregación de valor a la leche líquida o para la elaboración de derivados lácteos. El consumo de leche fluida sin procesamiento representó $29.2 \%$ del consumo total, mientras que el consumo para alimentación animal representó solo el $0.8 \%$ (FIRA, 2019). 
Internacionalmente India es el principal consumidor de leche fluida. Su demanda en 2018 representó el $26.7 \%$ de la producción mundial, adjudicada al crecimiento poblacional y al incremento en los ingresos de la población. A su vez, México ocupa el octavo lugar en el consumo de leche fluida; donde su demanda en el mismo año representó el 2.04\% de la producción mundial (FIRA, 2019) (figura 4).

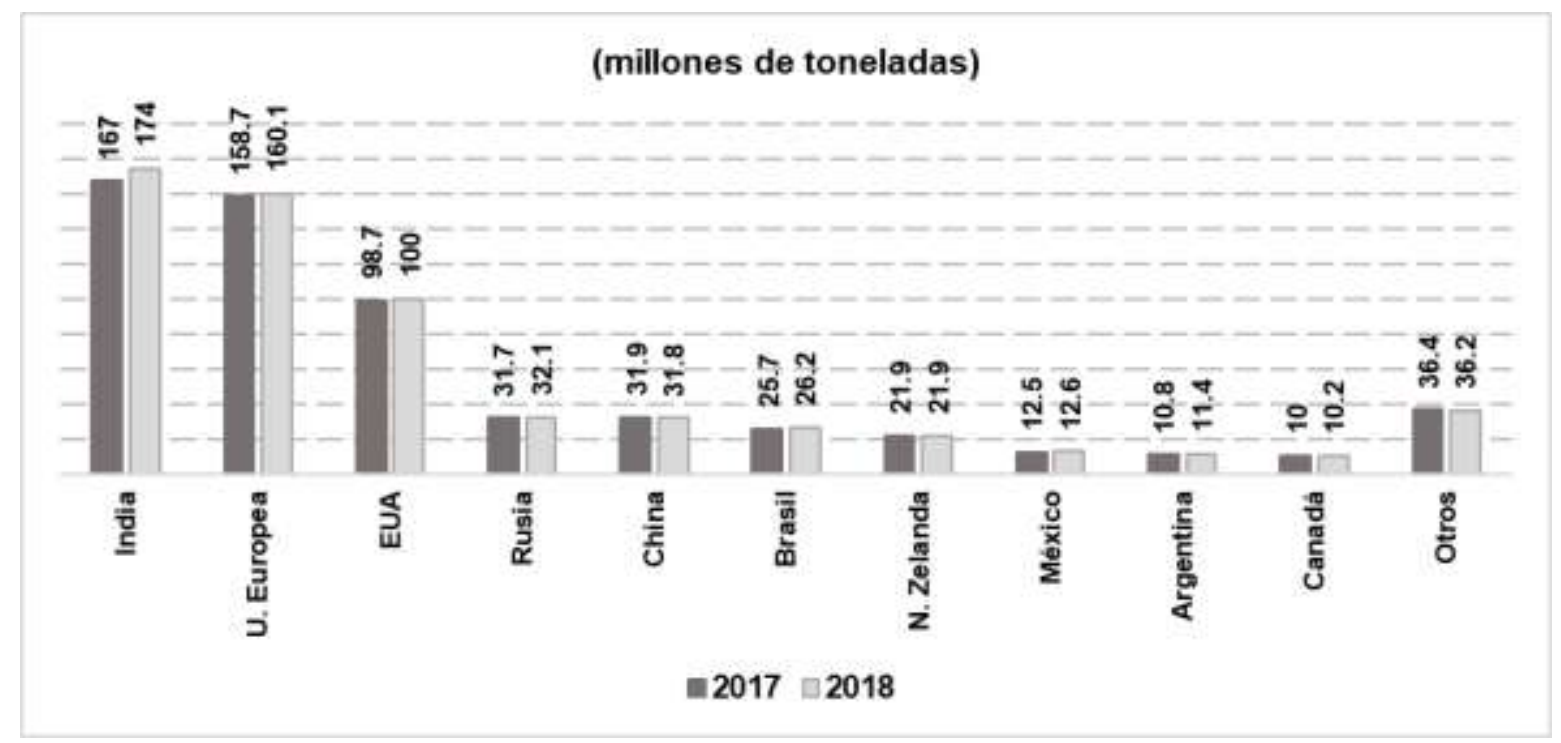

Fuente: FIRA con datos de la USDA, 2019.

Figura 4. Principales países consumidores de leche

Ahora bien, al realizar el análisis de correlación de Pearson y tomando como referencia a Hinkle et al. (2003) para su interpretación, se obtuvo una ausencia de correlación entre el consumo per cápita y las demás variables. Concluyendo así que las importaciones de leche en polvo al país (-.071) no influyen en el consumo de productos lácteos. Aunque es de importancia mencionar que el comportamiento del consumo per cápita en los últimos 10 años se mantiene al alza (SIAP, 2019). Pues autores como Montaño et al. (2012), mencionan que al norte de México los consumidores prefieren leche con grasa entera, donde la información proporcionada por el productor condicionará su consumo (Valencia et al., 2015).

\section{Importaciones de leche en polvo}

Aunque el sector lechero mexicano cuente con las condiciones ambientales favorables para ser productivo, las importaciones de leche en polvo actualmente se mantienen al alza. En este estudio, al realizar el análisis de correlación de Pearson y tomando como referencia a Hinkle et al. (2003) para su interpretación, se obtuvo como resultado una 
correlación alta entre el promedio de volumen anual (.883), coincidiendo con Engler y Nahuelhual (2003) quienes afirman que la leche importada y la producción nacional se comportan como sustitutas en el consumo y en la industria. De igual manera la variable Número de cabezas de ganado mantuvo una correlación alta (.822); lo cual resulta difícil de explicar. Pues se esperaría que, a mayor número de cabezas de ganado, la productividad también iría al alza y se esperaría así que las importaciones fueran menores. Mientras que la variable Consumo per cápita arrojó una ausencia de correlación (-.071).

A su vez, la variable precio constante mantiene una correlación moderada (.628), donde autores como Díaz et al. (2007) mencionan que "en el mercado de leche la industria ejerce un poder monopsónico sobre los precios y para evitar la caída de los precios nacionales se deben frenar las importaciones y ejercer controles sobre la industria". En la figura 5 se observa un crecimiento del precio constante y una tendencia a crecer de las importaciones de leche en polvo, pero se presume que existen otras causas de dependencia.

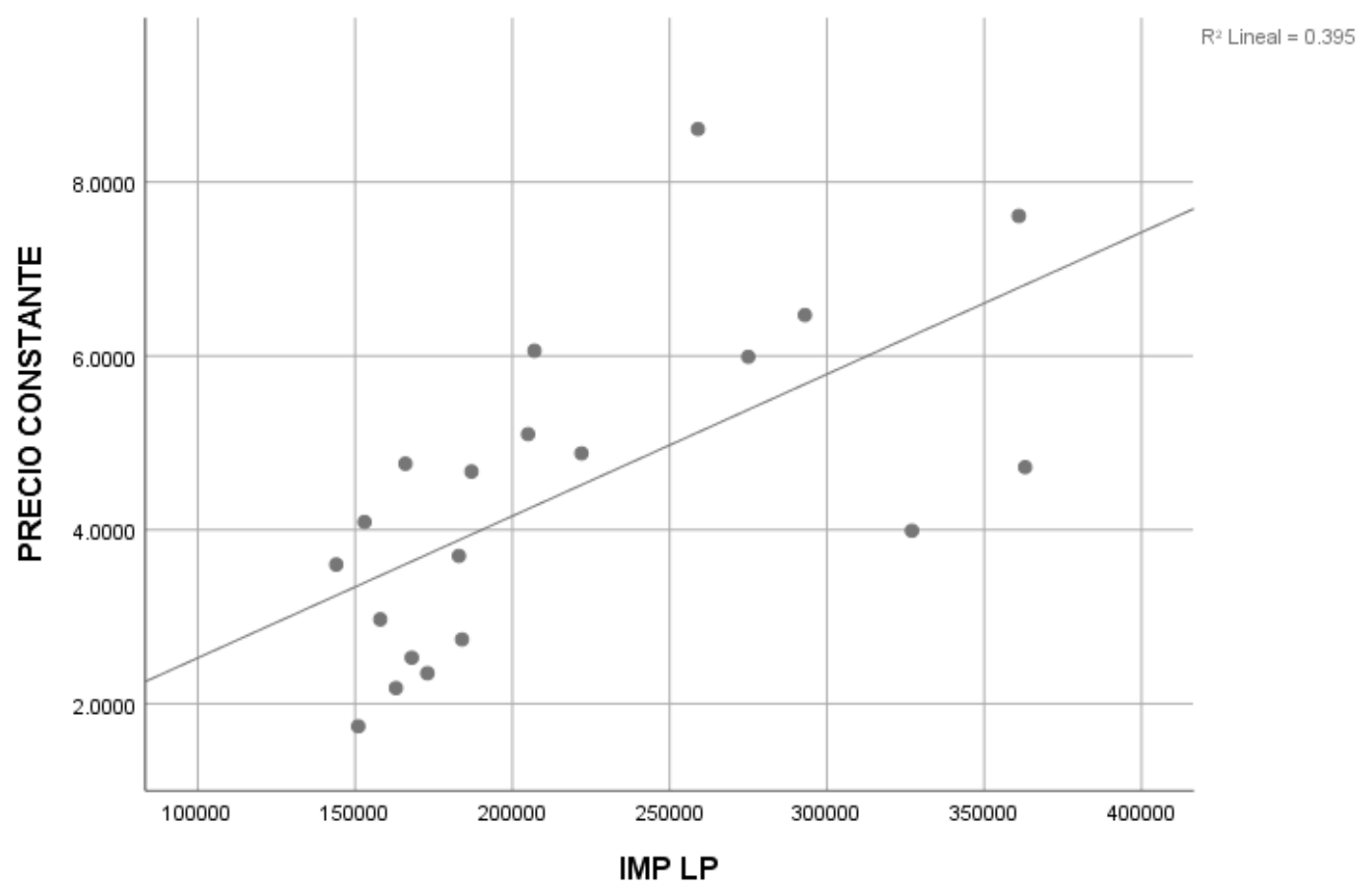

Fuente: Elaboración propia, con datos del SIAP (2020).

Figura 5. Dispersión simple con ajuste de línea de Precio constante por Importaciones de leche en polvo

Autores como Espinoza et al. (2019), mencionan que de continuar con la importación de leche en polvo y derivados se continuará afectando el precio de la leche fresca en el territorio nacional, lo cual, si consideramos la variabilidad en el precio constante 
observando en la figura 5, esta se puede adjudicar hasta cierto punto a las importaciones de leche en polvo; coincidiendo así con el autor anteriormente mencionado. Donde la leche en polvo importada de Estados Unidos entra al país a precios parcialmente bajos, aunque es de importancia el señalar que las importaciones solo representan $21 \%$ del mercado de leche (Ramírez et al., 2010). Siendo así México dependiente del mercado externo al importar más leche en polvo de la leche fresca que exporta (Rodríguez y Armenta, 2018).

\section{CONCLUSIÓN}

Se evaluó el contexto actual de la producción lechera mexicana tomando como referencia datos de las últimas dos décadas; a través de las variables cuantitativas: volumen, cabezas de ganado, precio constante, consumo per cápita e importaciones de leche en polvo. Donde los antecedentes mencionan que, a través del tiempo México no ha logrado mejorar su producción y competitividad en el ámbito internacional, a pesar de que el país cuenta con las condiciones climáticas ideales para la producción lechera. En el análisis de correlación realizado, se concluye que las importaciones de leche en polvo mantienen una alta correlación con la variable volumen; entendiéndose así que a pesar de que la producción se mantenga al alza, no es suficiente para abastecer la demanda nacional.

\section{LITERATURA CITADA}

ARCE BA, Martínez A. 2007. La cultura organizacional y de calidad como elementos básicos en la transformación de las empresas rurales hacia la competitividad. TECSISTECATL Revista de Economía y Sociedad de México. 1(2):1-21. ISSN: 18868452. https://www.eumed.net/rev/tecsistecatl/n2/acmd.htm

BACCO R, Valdivia C, Carranza A, Cámara R, Zavala P, Ancira E, Espinosa J. 2014. Brechas de rentabilidad económica en pequeñas unidades de producción de leche en el altiplano central mexicano. Revista mexicana de ciencias pecuarias. 5(3):273-290. ISSN:2007-1124. http://www.scielo.org.mx/scielo.php?script=sci_arttext\&pid=S200711242014000300002

BACHERO NJ, Blasco BO, Coll SV, Diez GR, Esteban GJ, Ivars EA, Ruiz PF. 2006. Estadística descriptiva y nociones de probabilidad. Editorial Paraninfo. Pp.434. ISBN:978-84973-23741

https://books.google.com.mx/books/about/Estad\%C3\%ADstica_descriptiva_y_nociones _de_p.html?id=8LS4Fa0JxRkC\&printsec=frontcover\&source=kp_read_button\&redir_esc $=\mathrm{y} \# \mathrm{v}=$ onepage \&q\&f=false

BARRIOS HD, Olivera ÁO. 2013. Análisis de la competitividad del sector lechero: caso aplicado al norte de Antioquia, Colombia. 23(48):33-41. ISSN:0121-5051. https://www.redalyc.org/pdf/818/81828690004.pdf 
CAMACHO VJ, Cervantes EF, Palacios RM, Rosales NF, Vargas CJ. 2017a. Factores determinantes del rendimiento en unidades de producción de lechería familiar. Revista Mexicana de Ciencias Pecuarias. 8(1):23-29. ISSN:2007-1124. http://www.scielo.org.mx/scielo.php?pid=S2007-11242017000100023\&script=sci_arttext CAMACHO VJ, Cervantes EF, Palacios RM, Cesín VA, Ocampo LJ. 2017b. Especialización de los sistemas productivos lecheros en México: la difusión del modelo tecnológico Holstein. Revista Mexicana de Ciencias Pecuarias. 8(3):259-268. ISSN: 2007-1124. http://www.scielo.org.mx/scielo.php?pid=S200711242017000300259\&script=sci_abstract\&tIng=en

CERVANTES EF, Cesín VJ. 2019. Análisis de la producción lechera e importaciones de lácteos en México ante la renegociación del TLCAN. La ganadería ante escenarios complejos. Universidad Autónoma Chapingo. Pp.15-27. ISBN:978-607-12-0571-1. https://www.researchgate.net/profile/Victor_Santos5/publication/340514478_Efectos_de _la_politica_pecuaria_en_el_sector_productivo_mexicano_El_caso_del_PROGAN_200 8-2013/links/5e8e139b299bf13079862e20/Efectos-de-la-politica-pecuaria-en-el-sectorproductivo-mexicano-El-caso-del-PROGAN-2008-2013. pdf\#page $=16$

CORDERA R, Heath J, Ibarra C, Ibarra D, Loría E. 2018. La economía mexicana: balance del 2017, principales tendencias, desafíos, y problemas de política económica. Revista de Economía 3:1-51. http://www.economia.unam.mx/assets/pdfs/econmex/03/01Ros.pdf

DÁVALOS FJ, Villegas VE. 2005. Análisis de razones financieras en la empresa lechera intensiva: Un estudio de caso en el altiplano mexicano. Veterinaria México. 36(1):153159. https://agris.fao.org/agrissearch/search.do?recordID=DJ2012035932

DFUS (Dairy Farming in the United States). 2020. Dairy Export Council. https://www.usdec.org/research-and-data/research-reports\#Scientific\%20Reports.

DÍAZ N, Melo O, Modrego F. 2007. Dinámica de transmisión de precios y cambio estructural en el sector lácteo chileno. Economía Agraria. 11(12):12-23. ISSN: 07189141. https://econpapers.repec.org/article/agseaaeac/97364.htm

DOPORTO M, Michelena G. 2011. La volatilidad de los precios de los commodities: el caso de los productos agrícolas. Revista del CEI. 19:35-56. ISSN: 1850-1737. https://dialnet.unirioja.es/servlet/articulo? codigo $=4080362$

ENGLER A,y Nahuelhual L. 2003. Influencia del mercado internacional de lácteos sobre el precio nacional de la leche: un análisis de cointegración. Agricultura Técnica. 63(4):416-427. ISSN: 0365-2807. http://dx.doi.org/10.4067/S0365-28072003000400010 
ESCUDERO C, Álvarez C, Irimia C. 2012. La necesidad del análisis de costes para mejorar la eficiencia en las explotaciones de vacuno lechero. Spanish Journal of Rural Development. 2:17-26. ISSN:2171-1216.

http://eds.a.ebscohost.com/abstract?site=eds\&scope=site\&authtype=crawler\&jnl=2171 1216\&asa $=Y \& A N=79907289 \& h=p P z O 7 g c V \% 2 f y T m r k R v d T t 4 b M y s M H G l x S u w c B i p W p 9 v$ b3\%2bueEG3MOTG2Dds0EGzdFzJD3kuDzltoh0bZL9MZ3fDQg\%3d\%3d\&crl=c\&resultL ocal=ErrCrlNoResults\&resultNs=Ehost\&crlhashurl=login.aspx\%3fdirect\%3dtrue\%26profi le\%3dehost\%26scope\%3dsite\%26authtype\%3dcrawler\%26jrnl\%3d21711216\%26asa\% 3dY\%26AN\%3d79907289

ESCUDERO C, Irimia S, Álvarez C. 2013. Análisis de costes. Gestión técnico económica de las explotaciones lecheras como herramienta de asesoramiento. Spanish Journal of Rural Development. 4:29-41. ISSN:2171-1216. http://eds.a.ebscohost.com/abstract?site=eds\&scope=site\&authtype=crawler\&jnl=2171 1216\&AN=89123276\&h=Ya79aGz2DB3OK8URkDGQ|Qum\%2fS\%2fkL2Rt90JxrB99W9 2NWUGAqXky 15lfhnlhHqcFd1\%2fp06SsxvcsEBRgECQE\%2bA\%3d\%3d\&crl=c\&resultL ocal=ErrCrlNoResults\&resultNs=Ehost\&crlhashurl=login.aspx\%3fdirect\%3dtrue\%26profi le\%3dehost\%26scope\%3dsite\%26authtype\%3dcrawler\%26jrnl\%3d21711216\%26AN\%3 d89123276

ESPINOZA AJ, Fabela HA, López CS, Martínez GF. 2019. Impacto de las importaciones de leche en polvo y derivados lácteos en el precio al productor de leche de bovino en México. Agricultura, sociedad y desarrollo. 16(1):123-139. ISSN:1870$5472 \quad \mathrm{http}: / /$ www.scielo.org.mx/scielo.php?script=sci_arttext\&pid=S187054722019000100123

FAO (Food and Agriculture Organization of the the United Nations). 2020. Producción lechera. Italia. http://www.fao.org/dairy-production-products/production/es/

FAO, Bioversity, CGIAR Consortium, FAO, IFAD, IFPRI, IICA, OECD, UNCTAD, Coordination Team of UN High Level Task Force on the Food Security Crisis, WFP, World Bank y WTO. 2012. Sustainable agricultural productivity growth and bridging the gap for small-family farms. Francia. Pp.89. http://www.fao.org/3/a-bt681e.pdf

FIRA (Fideicomisos Instituidos en Relación con la Agricultura). 2019. Leche y Lácteos 2019. México. Pp.26. https://www.inforural.com.mx/wpcontent/uploads/2019/06/Panorama-Agroalimentario-Leche-y-la769cteos-2019.pdf

GARAY AV, Sangerman JD, Rindermann RS, Vargas GA, Barrera JL. 2011. Determinación de la competitividad del sector agropecuario en México, 1980-2009. Revista Mexicana de Ciencias Agrícolas. 2(4):501-514. ISSN: 2007-9230. https://www.redalyc.org/pdf/2631/263119723003.pdf 
GIRÓN A. 2016. Objetivos del desarrollo sostenible y la agenda 2030: Frente a Las Políticas públicas y los cambios de gobierno en américa latina. Problemas del desarrollo. 47(186):3-8. ISSN:0301-7036.

http://www.scielo.org.mx/scielo.php?script=sci_arttext\&pid=S0301-70362016000300003

GUEVARA VG, Guevara VR, Pedraza OR, Fernández PN, Morales LA. 2004. Descripción de las unidades de producción lechera de la cuenca Camagüey-Jimaguayú, Cuba. Revista de Producción Animal. 16(1):9-16. ISSN: 0258-6010. https://core.ac.uk/download/pdf/268092798.pdf

HANSON J, Johnson D, Lichtenberg E, Minegishi K. 2013. Competitiveness of management-intensive grazing dairies in the mid-Atlantic region from 1995 to 2009. Journal of Dairy Science. 96(3):1894-1904. ISSN:0022-0302. https://doi.org/10.3168/jds.2011-5234

HEMME T, Uddin MM y Ndambi OA. 2014. Benchmarking cost of milk production in 46 countries. Journal of Reviews on Global Economics. 3:254-270. ISSN:1929-7092. http://www.lifescienceglobal.com/pms/index.php/jrge/article/view/1977/pdf_9

HERRERA RC, Méndez RC, Múnera BO, Cerón MM. 2011. Análisis de costos e ingresos de un hato lechero como herramienta para la toma de decisiones en el largo plazo. Revista Colombiana de Ciencias Pecuarias. 24:401-401. ISSN:0120-0690. https://revistas.udea.edu.co/index.php/rccp/article/view/324701/20782057

HINKLE DE, Wiersma W, Jurs SG. 2003. Applied statistics for the behavioral sciences. Massachusetts, USA: Houghton Mifflin College Division. Pp.756. ISBN:97806181-24053. https://www.worldcat.org/title/applied-statistics-for-the-behavioralsciences/oclc/643936092

JIMÉNEZ RA, Ortiz VE, Fonseca DM. 2014. El costo de oportunidad de la mano de obra familiar en la economía de la producción lechera de Michoacán, México. Revista de Investigación Agraria y Ambiental.5(1):47-56. ISSN: 2145-6097. https://hemeroteca.unad.edu.co/index.php/riaa/article/view/908

LOERA J, Banda J. 2017. Industria lechera en México: parámetros de la producción de leche y abasto del mercado interno. Revista de Investigaciones Altoandinas. 19(4):419426. ISSN: 2313-2957. http://www.scielo.org.pe/scielo.php?pid=S231329572017000400008\&script=sci_arttext\&tlng=pt 
LÓPEZ G, Espinoza J, Espinosa J, Jolalpa J, Vélez A. 2014. Variables del contexto nacional e internacional en los sistemas de producción lecheros mexicanos. Agrofaz. 14(1):15-31. ISSN: 1665-8892.

https://www.researchgate.net/profile/Jose_Espinoza7/publication/312469057_Variables _del_contexto_nacional_e_internacional_en_los_sistemas_de_produccion_lecheros_m exicanos/links/5ab28e04a6fdcc1bc0c1e2b0/Variables-del-contexto-nacional-einternacional-en-los-sistemas-de-produccion-lecheros-mexicanos.pdf

MAIN MV. 2002. Factores que inciden en la satisfacción laboral del obrero rural. Revista Interamericana de Psicología Ocupacional. 21(1).28-37. ISSN: 2539-5238. http://revista.cincel.com.co/index.php/RPO/article/view/41

MAMIAN CA, Erazo XA, Velasco SM. 2016. Huella hídrica de una finca ganadera lechera bajo las condiciones agroecológicas del Valle del Cauca. Biotecnología en el Sector Agropecuario y Agroindustrial: BSAA. 14(2):47-56. ISSN: 1692-3561. https://dialnet.unirioja.es/servlet/articulo?codigo $=6117858$

MONTAÑo MI, Avendaño RB, Acosta MA, Mesías DF. 2013. Preferencias de leche fresca de bovino del consumidor de Baja California, México. Revista Mexicana de Ciencias Pecuarias. 4(1):47-60. ISSN:2007-1124.

http://www.scielo.org.mx/scielo.php?pid=S2007-11242013000100004\&script=sci_arttext

NÚÑEZ JM. 2016. Productividad, comercialización y calidad de vida en los productores lecheros de la Ciénega de Jalisco, México. Revista Mexicana de Agronegocios. 38:367376. ISSN:1405-9282.

https://www.redalyc.org/jatsRepo/141/14146082013/html/index.html

OIE (Organización Mundial de Sanidad Animal). 2013. Código Sanitario para los Animales Terrestres. $\quad$ Francia. $\quad$ Pp.712. http://www.oie.int/index.php?id=169\&L=2\&htmfile=chapitre_1.7.7.htm

OIE (Organización Mundial de Sanidad Animal). 2014. Bienestar Animal y Sistemas de Producción De Vacas Lecheras. Francia. 281-322p. https://www.oie.int/fileadmin/Home/esp/Internationa_Standard_Setting/docs/pdf/E_TAH SC_Feb_2014_Parte_B.pdf

OLIVERA J, García M, Parra RC. 2018. Impacto del manejo administrativo y su trascendencia sobre el concepto de desarrollo sustentable en las explotaciones agropecuarias de la región norte del estado de Jalisco. NovaRua. 8(15):102-112. ISSN:2007-4042. http://erevistas.uacj.mx/ojs/index.php/NovaRua/article/view/2344 
PRÓSPERO F, Martínez A, Pérez J, Arriaga C, Vicente F. 2013. Mejora en la rentabilidad de la producción lechera a partir del uso de ensilado de maíz cultivado con fertilización orgánica. AIDA XV Jornadas sobre Producción Animal. 1:28-30. https://www.aida-itea.org/aidaitea/files/jornadas/2013/comunicaciones/2013_SGEG_04.pdf

QÜESTA T, Quagliani A, López R. 2016. La asimétrica traslación de precios en la cadena láctea: una interpretación teórica. Revista de Investigaciones de la Facultad de Ciencias Agrarias-UNR. (08):15-27. ISSN: 1853-4333.

https://cienciasagronomicas.unr.edu.ar/journal/index.php/agronom/article/view/176

RAMíREZ JR, García SJ, García MR. 2010. Efectos del tratado de libre comercio de América del Norte sobre la producción de leche en México. Universidad y Ciencia. 26(3):283-292. ISSN:0186-2979.

http://www.scielo.org.mx/scielo.php?script=sci_arttext\&pid=S0186-29792010000300007

ROBLEDO PR. 2018. Producción de Leche en México y el Impacto de las Importaciones de Leche en Polvo. Universidad Nacional Autónoma de México y Asociación Mexicana de Ciencias para el Desarrollo Regional. México. Pp. 2016-224. ISBN:978-607-30-0970-6. http://ru.iiec.unam.mx/4223/1/2-Vol1_Parte2_Eje2_Cap2-192Robledo.pdf

RODRÍGUEZ SJ, Ramírez AB. 2018. Panorama sobre la producción y el consumo de leche y lácteos en México. Hitos de Ciencias Económico Administrativas. 24(70):518534. ISSN:2448-4733. https://revistas.ujat.mx/index.php/hitos/article/view/2949/2269

SAGARPA (Secretaría de Agricultura, Ganadería, Desarrollo Rural, Pesca y Alimentación). 2004. Situación actual de la producción de leche de bovino en México $2004 . \quad$ México. Pp. 42. http://www.lactodata.info/docs/lib/sagarpa_cgg_situacion_actual_produccion_2004.pdf

SALOMÓN JM, Ramírez AB. 2018. Panorama sobre la producción y el consumo de leche y lácteos en México. Hitos de Ciencias Económico Administrativas. 24(70):518534. ISSN:2448-4733. https://revistas.ujat.mx/index.php/hitos/article/view/2949

SANTIBÁÑEZ SJ, Sánchez AM. 2009. Transnacionalización del mercado de lácteos y vía nacional del desarrollo. Los casos de México y Japón. Revista Polis. 5(2):155-200. ISSN:1870-2333. http://www.scielo.org.mx/pdf/polis/v5n2/v5n2a6.pdf

SEGURA CU, Lozano JL. 2015. Evaluación de unidades familiares de producción lechera en Aguascalientes: estrategias para incrementar su producción y rentabilidad. Avances en Investigación Agropecuaria. 19(2):7-34. ISSN:0188-7890. https://www.redalyc.org/pdf/837/83742619002.pdf 
SIAP (Servicio de Información Agroalimentaria y Pesquera). 2019. Boletín de Leche (enero-marzo 2019). México. Pp. 78.

http://infosiap.siap.gob.mx/opt/boletlech/Bolet\%C3\%ADn\%20de\%20Leche\%20eneromarzo\%202019.pdf

SRAÏRI TM, Tahar BM, Kraiem K. 2013. The dairy chains in North Africa (Algeria, Morocco and Tunisia): from self sufficiency options to food dependency?. SpringerPlus. 2(162):1-13. ISSN: 2193-1801. https://doi.org/10.1186/2193-1801-2-162

TORSTEN H, García O, Saha A. 2003. La producción de leche en la india, oportunidades y riesgos para los pequeños productores. Italia. Pp.2. http://www.fao.org/tempref/AG/Reserved/PPLPF/Docs/Project\%20Publications/Policy\% 20Briefs/Policy\%20briefs\%20x\%20printing/PB_WP02/02_esp.pdf

VALENCIA SK, Flores JS, Paz JD, Damián MÁ, Huerta HV. 2015. Factores que determinan el consumo de leche en el Distrito Federal, México. Revista Científica. 25(1):74-80. ISSN:0798-2259.

https://www.redalyc.org/pdf/959/95934122011.pdf

ZÁRATE MJ, Esqueda EV, Vinay VJ, Jácome MS. 2010. Evaluación económicoproductiva de un sistema de producción de leche en el trópico. Agronomía mesoamericana. 21(2):255-265. ISSN:1659-1321.

https://www.scielo.sa.cr/scielo.php?script=sci_arttext\&pid=S1659-13212010000200004 